\section{Home Vaccination - The way forward in a Pandemic}

When the coronavirus disease 2019 (COVID-19) pandemic struck in early 2020, the associated lockdowns and the fear of contracting the disease made parents miss their children's vaccinations. India saw a $70 \%$ reduction in routine vaccinations during early 2020 , and concerns were expressed by World Health Organization (WHO) and United Nation's (UNICEF) that vaccine preventable disease (VPD) may make a comeback [1,2]. In this background, we introduced of home vaccinations [3]. We offered home vaccinations to parents who were afraid of coming out or could not come out due to various reasons.

The criteria for offering home vaccination were: parents to consult a pediatrician on video consultation or tele consultation - to discuss the vaccinations that are due there for child [4]; the pediatrician after detailed history would explain the vaccinations and possible common side effects and prescribe the vaccinations; and this prescription then be handled by a qualified pharmacist who will dispense the vaccines. The vaccines were delivered by a team of two experienced nurses, an ambulance officer and an ambulance driver capable of handling emergencies like anaphylaxis. This team visited the family in appropriate personal protective equipment (PPE), match the child's details with the prescription and administer the vaccines after taking parental consent. The team had provision of all emergency drugs, and observed the child for half an hour for any possible side effects.

This concept of home vaccination has gained good acceptance. In 2020, we carried out more than 25,000 home vaccinations for children. We feel that this concept is worth taking up on a wider scale by hospitals and non-governmental organizations so as to address the possibility of resurgence of VPDs during this pandemic.

\section{REFERENCES}

R KISHORE KUMAR Cloudnine Hospital, Bangalore, Karnataka. drkishore@cloudninecare.com

1. GAVI Alliance. Vaccinations during a pandemic: Benefit or risk? Accessed June 10, 2021. Available from: https://www. gavi.org/vaccineswork/routine-vaccinations-during-pandemic-benefit-or-risk? gclid $=C j w K C A j w 7$ diEBhB-EiwAsk Vi10Gf1ZTiJKM5SBo6jfOwuI43gNeCkLVAOpF7kxXL8S-HF AbkySaydBoCJugQAvD BwE

2. Khatiwada AP, Shrestha N, Shrestha S. Will Covid-19 lead to a resurgence of vaccine preventable diseases? Accessed June 10, 2021. Available from: https://www.dovepress.com/willcovid-19-lead-to-a-resurgence-of-vaccine-preventable-diseases-peer-reviewed-fulltext-article-IDR

3. Ogbuanu IU, Li AJ, Anya BM, et al. Can vaccination coverage be improved by reducing missed opportunities for vaccination? Findings from assessments in Chad and Malawi using the new WHO methodology. PLoS One. 2019;14:e0210648.

4. Ames HMR, Glenton C, Lewin S, Cochrane Consumers and
Communication Group. Parents' and informal caregivers' views and experiences of communication about routine childhood vaccination: A synthesis of qualitative evidence. Cochrane Database Syst Rev. 2017;2017:CD011787.

\section{Unusual Complication of Hair Pulling in a Young Child}

A 7-year-old girl, who was previously well, presented with complaints of scalp swelling. Two days prior to presentation, she had pain during combing of hairs, when her mother noticed swelling over the scalp and the swelling gradually increased in size over the next two days. There was no history of trauma, or family history suggestive of either bleeding disorder or coagulopathy. She was not on any medications, and had not undergone any surgical procedures.

On examination, she was conscious, alert, and had stable vitals. Neurologic examination and other systemic examinations were unremarkable. Local examination of the scalp revealed diffuse nontender boggy swelling over the left parietal area. Skin over the swelling was normal. Computed tomography (CT) of head showed subgaleal bleed without skull fracture and intracranial pathology. Complete blood count, coagulation profile, factor XIII and VWF levels were normal.

As the child was hemodynamically stable, no neurosurgical intervention was required. Parents were counselled regarding the possible complications such as infections, calcification and further bleeding. The swelling was noted to decrease in size during follow up.

Subgaleal hematomas occurring beyond the neonatal period are rare and usually secondary to significant head trauma. Either tangential (blunt) or radial (pulling) forces cause shearing and rupture of emissary veins traversing the subgaleal space. Subgaleal hematoma has been reported following relatively minor trauma such as hair braiding or sudden hair pulling [1-3]. They are usually small, self-limiting in nature, remain localized, and resolve spontaneously and are usually conservatively managed. As this child presented with significant swelling, coagulation abnormality was considered as there was no history of trauma. As her investigations were unremarkable and she was hemodynamically stable, she was treated conservatively and the swelling resolved completely within two weeks. Edmondson, et al. [4] have previous reported a patient with delayed presentation of a massive subgaleal hematoma in an adolescent following a seemingly innocuous episode of hair pulling, in the absence of underlying hematological or anatomical abnormality [3].

In conclusion, although subgaleal bleed beyond neonatal period is rare, it can happen and the patient has to be investigated with neuroimaging to look for the skull fracture and hematoma extension, and investigated to rule out rare coagulation disorders, as subgaleal bleed due to hair pulling is a diagnosis of exclusion. 